\title{
Article
}

\section{On a Conjecture about the Sombor Index of Graphs}

\author{
Kinkar Chandra Das ${ }^{1, *} \mathbb{0}$, Ali Ghalavand ${ }^{2}$ and Ali Reza Ashrafi ${ }^{2}$ \\ 1 Department of Mathematics, Sungkyunkwan University, Suwon 16419, Korea \\ 2 Department of Pure Mathematics, Faculty of Mathematical Sciences, University of Kashan, \\ Kashan 87317-53153, Iran; alighalavand@grad.kashanu.ac.ir (A.G.); ashrafi@kashanu.ac.ir (A.R.A.) \\ * Correspondence: kinkardas2003@gmail.com
}

Citation: Das, K.C.; Ghalavand, A.; Ashrafi, A.R. On a Conjecture about the Sombor Index of Graphs. Symmetry 2021, 13, 1830. https:// doi.org/10.3390/sym13101830

Academic Editors: Jose M. Rodriguez and Eva Tourís

Received: 19 June 2021

Accepted: 2 September 2021

Published: 1 October 2021

Publisher's Note: MDPI stays neutral with regard to jurisdictional claims in published maps and institutional affiliations.

\begin{abstract}
Let $G$ be a graph with vertex set $V(G)$ and edge set $E(G)$. A graph invariant for $G$ is a number related to the structure of $G$ which is invariant under the symmetry of $G$. The Sombor and reduced Sombor indices of $G$ are two new graph invariants defined as $S O(G)=\sum_{u v \in E(G)} \sqrt{d_{G}(u)^{2}+d_{G}(v)^{2}}$ and $S O_{r e d}(G)=\sum_{u v \in E(G)} \sqrt{\left(d_{G}(u)-1\right)^{2}+\left(d_{G}(v)-1\right)^{2}}$, respectively, where $d_{G}(v)$ is the degree of the vertex $v$ in $G$. We denote by $H_{n, v}$ the graph constructed from the star $S_{n}$ by adding $v$ edge(s), $0 \leq v \leq n-2$, between a fixed pendent vertex and $v$ other pendent vertices. Réti et al. [T. Réti, T Došlić and A. Ali, On the Sombor index of graphs, Contrib. Math. 3 (2021) 11-18] proposed a conjecture that the graph $H_{n, v}$ has the maximum Sombor index among all connected $v$-cyclic graphs of order $n$, where $0 \leq v \leq n-2$. In some earlier works, the validity of this conjecture was proved for $v \leq 5$. In this paper, we confirm that this conjecture is true, when $v=6$. The Sombor index in the case that the number of pendent vertices is less than or equal to $n-v-2$ is investigated, and the same results are obtained for the reduced Sombor index. Some relationships between Sombor, reduced Sombor, and first Zagreb indices of graphs are also obtained.
\end{abstract}

Keywords: Sombor index; reduced Sombor index; first Zagreb index; extremal problem

MSC: 05C07; 05C09; 05C35

\section{Basic Definitions}

Throughout this paper, all graphs considered are finite, undirected, and simple. Let $G$ be such a graph with vertex set $V=V(G)$ and edge set $E=E(G)$. We recall that the degree of a vertex $v$ in $G, d_{G}(v)$, is defined as the number of edges incident to $v$. Let $\Delta$ be the maximum degree in $G$. The set of all vertices adjacent to the vertex $v$ is denoted by $N(G, v)$. The edge degree of $e \in E(G)$ is the degree of $e$ in the line graph of $G$. If we define $\varepsilon_{i}=\varepsilon_{i}(G)$ to be the number of edges of degree $i$ in $G$, then it can be easily seen that $\sum_{i=0}^{2 \Delta(G)-2} \varepsilon_{i}=|E(G)|$. We also use the notation $m_{i, j}(G)$ for the number of edges of $G$ with endpoints of degrees $i$ and $j$.

A graph $G$ with this property that the degree of each vertex is at most four is called a chemical graph. Suppose $V(G)=\left\{v_{1}, v_{2}, \ldots, v_{n}\right\}$ and $d_{G}\left(v_{1}\right) \geq d_{G}\left(v_{2}\right) \geq \cdots \geq d_{G}\left(v_{n}\right)$. Then, the sequence $d(G)=\left(d_{G}\left(v_{1}\right), d_{G}\left(v_{2}\right), \ldots, d_{G}\left(v_{n}\right)\right)$ is called the degree sequence of $G$. The graph union $G \cup H$ of two graphs $G$ and $H$ with disjoint vertex sets is another graph with $V(G \cup H)=V(G) \cup V(H)$ and $E(G \cup H)=E(G) \cup E(H)$. The union of $s$ disjoint copies is denoted by $s G$. The join $G+H$ of two graphs $G$ and $H$ with disjoint vertex sets is a graph with vertex set $V(G \cup H)$ and edge set $E(G \cup H) \cup\{u v \mid u \in V(G) \wedge v \in V(H)\}$.

Suppose $f$ is an edge and $u, v$ are two non-adjacent vertices of $G$. Then, $G-f$ is the subgraph of $G$ obtained by deleting the edge $f$ and $G+u v$ is a graph obtained from $G$ by adding an edge connecting $u$ and $v$.

Suppose $G$ is a connected graph with exactly $n$ vertices and $m$ edges. The cyclomatic number of $G$ is defined as $v(G)=m-n+1$ and a $v$-cyclic graph is a graph with cyclomatic 
number $v$. For terms and notations not defined here, we follow the standard texts in graph theory as the famous book of West [1].

A graph invariant for $G$ is a number related to the structure of $G$ which is invariant under the symmetry of $G$. The first Zagreb index of a graph $G$ is an old degree-based graph invariant introduced by Gutman and Trinajstić [2] defined as $M_{1}(G)=\sum_{u v \in E(G)}\left[d_{G}(u)+\right.$ $\left.d_{G}(v)\right]=\sum_{v \in V(G)} d_{G}(v)^{2}$. In a recent paper about the general form of all degree-based topological indices of graphs [3], Gutman introduced two new invariants and invited researchers to investigate their mathematical properties and chemical meanings. He used the names "Sombor index" and "reduced Sombor index" for his new graphical invariants. The Sombor and reduced Sombor indices are defined as follows:

$$
\begin{aligned}
S O(G) & =\sum_{u v \in E(G)} \sqrt{d_{G}(u)^{2}+d_{G}(v)^{2}}, \\
S O_{r e d}(G) & =\sum_{u v \in E(G)} \sqrt{\left(d_{G}(u)-1\right)^{2}+\left(d_{G}(v)-1\right)^{2}} .
\end{aligned}
$$

Very recently, Redžepović [4] discussed chemical applicability on the Sombor index of graphs. Specifically, the Sombor index was used to model entropy and enthalpy of vaporization of alkanes with satisfactory prediction potential, indicating that this topological index may be used successfully on modeling thermodynamic properties of compounds. In the same paper, the level of the predicting power of the Sombor index and its degeneracy were tested.

We refer to [5,6], for more information on degree-based topological indices of graphs and their extremal problems.

Let $\Gamma(s, n)$ denote the set of all decreasing real sequences $c=\left(c_{1}, c_{2}, \ldots, c_{n}\right)$ such that $\sum_{i=1}^{n} c_{i}=s$. Define a relation $\preceq$ on $\Gamma(s, n)$ as follows: For two decreasing real sequences $c=\left(c_{1}, c_{2}, \ldots, c_{n}\right)$ and $d=\left(d_{1}, d_{2}, \ldots, d_{n}\right)$ in $\Gamma(s, n)$, we write $c \preceq d$ if and only if for each integer $k, 1 \leq k \leq n-1$, we have $\sum_{i=1}^{k} c_{i} \leq \sum_{i=1}^{k} d_{i}$. It is easy to see that $(\Gamma(s, n), \preceq)$ is a partially ordered set. The partial order $\preceq$ is called the majorization and, if $c \preceq d$, then we say that $c$ is majorized by $d$. We refer the interested readers to consult the survey article [7] and the book [8], for more information on majorization theory and its applications in graph theory.

Suppose $X \subseteq \mathbb{R}^{n}$ and $a, b \in X$ are different points in $X$. The line segment $\overline{a b}$ is the set of all points $\lambda a+(1-\lambda) b$, where $0<\lambda<1$. The set $X$ is said to be convex, if for every point $a, b \in X, \overline{a b} \subseteq X$. Let $X \subseteq \mathbb{R}^{n}$ be convex. The function $f: X \longrightarrow \mathbb{R}$ is called a convex function, if for any $a, b \in X$ and $0<\lambda<1$, we have $f(\lambda a+(1-\lambda) b) \leq \lambda f(a)$ $+(1-\lambda) f(b)$. If $f$ is convex and we have strict inequality for all $a \neq b$, then we say the function is strictly convex. It is well-known that if $I$ is an open interval and $g: I \longrightarrow \mathbb{R}$ is a real twice-differentiable function on $I$, then $g$ is convex if and only if for each $x \in I$, $g^{\prime \prime}(x) \geq 0$. The function $g$ is strictly convex on $I$, if $g^{\prime \prime}(x)>0$ for all $x \in I$.

\section{Background Materials}

In [3], Gutman proved that, among all $n$-vertex graphs, the empty graph $\overline{K_{n}}$ and the complete graph $K_{n}$ have the minimum and maximum Sombor indices, respectively. He also proved that, if we restrict our attention to the $n$-vertex connected graph, then the $n$-vertex path $P_{n}$ will attain the minimum Sombor index. He also proved in [9] that $M_{1}(G) \geq S O(G) \geq \frac{1}{\sqrt{2}} M_{1}(G)$, and, if $G$ has $m$ edges, then $M_{1}(G)-2 m \geq S O_{\text {red }}(G) \geq$ $\frac{1}{\sqrt{2}}\left(M_{1}(G)-2 m\right)$.

Cruz et al. [10] characterized the extremal graphs with respect to the Sombor index over the set of all $n$-vertex chemical graphs, connected chemical graphs, chemical trees, and hexagonal systems. Cruz and Rada [11], studied the extremal values of Sombor index over the set of all unicyclic and also bicyclic graphs of a given order. In a recent work, Das et al. [12] obtained lower and upper bounds for the Sombor index of graphs based 
on some other graph parameters. Moreover, they obtained some relationships between Sombor index and the first and second Zagreb indices of graphs.

Deng et al. [13] investigated the chemical importance of the Sombor index and obtained the extremal values of the reduced Sombor index for chemical trees. Milovanović et al. [14] investigated the relationship between Sombor index and Albertson index which is an old irregularity measure for graphs, and, in [4], Redžepović examined the predictive and discriminative potentials of Sombor and reduced Sombor indices of chemical graphs. Wang et al. [15] investigated the relationships between the Sombor index and some degree based invariants, and obtained some Nordhaus-Gaddum type results. In [16,17], the authors presented some bounds on the Sombor index of some class of graphs in terms of graph parameters and characterized the extremal graphs. Ghanbari and Alikhani [18] computed this index for certain graphs and examined its effects under some operations on vertex and edge of $G$. As a consequence, they presented bounds for the Sombor index of the join and corona product of two graphs.

The following lemma [19] is useful in some of our results.

Lemma 1. Suppose $c=\left(c_{1}, c_{2}, \ldots, c_{n}\right)$ and $d=\left(d_{1}, d_{2}, \ldots, d_{n}\right)$ are two decreasing sequences of real numbers. If $c \preceq d$, then, for any convex function $f, \sum_{i=1}^{n} f\left(c_{i}\right) \leq \sum_{i=1}^{n} f\left(d_{i}\right)$. Furthermore, if $c \prec d$ and $f$ is a strictly convex function, then $\sum_{i=1}^{n} f\left(c_{i}\right)<\sum_{i=1}^{n} f\left(d_{i}\right)$.

The graph constructed from the star $S_{n}$ by adding $v$ edge(s), $0 \leq v \leq n-2$, between a fixed pendent vertex and $v$ other pendent vertices is denoted by $H_{n, v}$ [20].

Conjecture 1 (Réti et al. [20]). If $v$ and $n$ are fixed integers satisfying the inequality $5 \leq v \leq$ $n-2$, then, among all connected $v$-cyclic graphs of order $n$, only the graph $H_{n, v}$ has the maximum Sombor index.

\section{New Bounds on the Sombor Index}

The aim of this section is to present some new bounds on Sombor and reduced Sombor indices of graphs. The relationship between the Sombor index, reduced Sombor index, and the first Zagreb index of graphs will also be investigated.

Lemma 2. Let $G$ be an n-vertex connected graph with cyclomatic number $v$ and degree sequence $d(G)=\left(d_{G}\left(v_{1}\right), d_{G}\left(v_{2}\right), \ldots, d_{G}\left(v_{n}\right)\right)$ with this property that the number of pendent vertices is less than or equal to $n-v-2$. If $0 \leq v \leq n-2$ and $d_{G}\left(v_{1}\right)=n-1$, then

$$
\left(d_{G}\left(v_{2}\right), d_{G}\left(v_{3}\right), \ldots, d_{G}\left(v_{n}\right)\right) \preceq(v+1, \overbrace{2, \ldots, 2}^{v}, \overbrace{1, \ldots, 1}^{n-v-2}) .
$$

Proof. We have $v=m-n+1$. Moreover,

$$
\sum_{i=2}^{n} d_{G}\left(v_{i}\right)=2 m-d_{G}\left(v_{1}\right)=2 v+2 n-2-d_{G}\left(v_{1}\right)=2 v+n-1 .
$$

On the other hand, the sum of all integers on the right hand side of (1) is again $2 v+n-1$. Let $c=\left(c_{2}, \ldots, c_{n}\right)=\left(d_{G}\left(v_{2}\right), \ldots, d_{G}\left(v_{n}\right)\right)$ and let $d=\left(d_{2}, \ldots, d_{n}\right)=(v+$ $1,2, \ldots, 2,1, \ldots, 1)$, where the multiplicities of the numbers 1 and 2 in the last sequence are $n-v-2$ and $v$, respectively. Thus, we have

$$
\sum_{i=2}^{n} c_{i}=\sum_{i=2}^{n} d_{G}\left(v_{i}\right)=2 v+n-1=\sum_{i=2}^{n} d_{i}
$$

Since the number of pendent vertices is less than or equal to $n-v-2$ in $G$, one can easily see that $c_{i} \geq d_{i}$ for $i=3,4, \ldots, n$. From this result with (2), we conclude that $\sum_{i=2}^{k} c_{i} \leq \sum_{i=2}^{k} d_{i}(2 \leq k \leq n-1)$. Hence, $c \preceq d$. This completes the proof of the result. 
Lemma 3 (Réti et al. [20]). Suppose $G$ is a connected graph with a maximum Sombor index among all connected graphs with $n$ vertices and cyclomatic number $v$. If $0 \leq v \leq n-2$, then $\Delta(G)=n-1$.

$$
\text { Define } S O^{\ddagger}(G)=\sum_{u v \in E(G)} \sqrt{\left(d_{G}(u)+1\right)^{2}+\left(d_{G}(v)+1\right)^{2}} .
$$

Lemma 4. Let $G$ be a graph with $n$ vertices and $m$ edges. Then,

$$
S O^{\ddagger}(G) \leq m \sqrt{(m+1)^{2}+4} .
$$

For $m \leq n-1$, the equality holds if and only if $G \cong S_{m+1} \cup(n-m-1) K_{1}$.

Proof. For any $u v \in E(G)$, we have $d_{G}(u)+d_{G}(v) \leq m+1$. Note that the function $g(x)=(x+1)^{2}$ is strictly convex on $(-\infty, \infty)$. For any edge $u v \in E(G)$, we have

$$
\sqrt{\left(d_{G}(u)+1\right)^{2}+\left(d_{G}(v)+1\right)^{2}} \leq \sqrt{\left(d_{G}(u)+1+m+1-d_{G}(u)-d_{G}(v)\right)^{2}+\left(d_{G}(v)+1\right)^{2}}
$$

and $\left(d_{G}(u)+m+1-d_{G}(u)-d_{G}(v), d_{G}(v)\right) \preceq(m, 1)$. Moreover, the equality holds in (3) if and only if $d_{G}(u)+d_{G}(v)=m+1$. For any $u v \in E(G)$, by Lemma 1 , we have

$$
\sqrt{\left(d_{G}(u)+1+m+1-d_{G}(u)-d_{G}(v)\right)^{2}+\left(d_{G}(v)+1\right)^{2}} \leq \sqrt{(m+1)^{2}+4}
$$

with equality holding if and only if $\left(d_{G}(u)+m+1-d_{G}(u)-d_{G}(v), d_{G}(v)\right)=(m, 1)$. For any $u v \in E(G)$, we obtain

$$
\sqrt{\left(d_{G}(u)+1\right)^{2}+\left(d_{G}(v)+1\right)^{2}} \leq \sqrt{(m+1)^{2}+4}
$$

with equality holding if and only if $\left(d_{G}(u), d_{G}(v)\right)=(m, 1)$. Hence,

$$
\begin{aligned}
S O^{\ddagger}(G) & =\sum_{u v \in E(G)} \sqrt{\left(d_{G}(u)+1\right)^{2}+\left(d_{G}(v)+1\right)^{2}} \\
& \leq \sum_{u v \in E(G)} \sqrt{(m+1)^{2}+4} \\
& =m \sqrt{(m+1)^{2}+4} .
\end{aligned}
$$

Moreover, the above equality holds if and only if $G \cong S_{m+1} \cup(n-m-1) K_{1}$ as $m \leq n-1$.

Lemma 5. Let $n \geq 3$. The two functions $f(x)=\sqrt{(n-1)^{2}+x^{2}}$ and $g(x)=\sqrt{(n-2)^{2}+(x-1)^{2}}$ are strictly convex on $(-\infty, \infty)$.

Proof. We have $f^{\prime \prime}(x)=\frac{(n-1)^{2}}{\left(x^{2}+(n-1)^{2}\right)^{\frac{3}{2}}}$. Therefore, for each $r, r \in(-\infty, \infty)$, we have $f^{\prime \prime}(r)>0$. This proves that $f$ is strictly convex on $(-\infty, \infty)$. Similarly, one can easily prove that $g(x)=\sqrt{(n-2)^{2}+(x-1)^{2}}$ is strictly convex on $(-\infty, \infty)$.

Lemma 6. Let $G$ be a connected graph with cyclomatic number $v(0 \leq v \leq n-2)$ and vertex set $V(G)=\left\{v_{1}, v_{2}, \ldots, v_{n}\right\}$ with the condition that the number of pendent vertices is less than or equal to $n-v-2$. If $d(G)=\left(n-1, d_{G}\left(v_{2}\right), \ldots, d_{G}\left(v_{n}\right)\right)$; then, 


$$
\begin{gathered}
\sum_{v \in V(G) \backslash\left\{v_{1}\right\}} \sqrt{(n-1)^{2}+d_{G}(v)^{2}} \leq(n-v-2) \sqrt{(n-1)^{2}+1}+v \sqrt{(n-1)^{2}+4} \\
+\sqrt{(n-1)^{2}+(v+1)^{2}}
\end{gathered}
$$

with equality if and only if $G \cong H_{n, v}$.

Proof. Suppose $f(x)=\sqrt{(n-1)^{2}+x^{2}}$. By Lemma $5, f$ is strictly convex on $(-\infty, \infty)$. By Lemma $2,\left(d_{G}\left(v_{2}\right), d_{G}\left(v_{3}\right), \ldots, d_{G}\left(v_{n}\right)\right) \preceq(v+1,2, \ldots, 2,1, \ldots, 1)$, where the multiplicities of the numbers 1 and 2 in the last sequence are $n-v-2$ and $v$, respectively. Now, Lemma 1 implies that $\sum_{v \in V(G) \backslash\left\{v_{1}\right\}} \sqrt{(n-1)^{2}+d_{G}(v)^{2}} \leq(n-v-2) \sqrt{(n-1)^{2}+1}$ $+v \sqrt{(n-1)^{2}+4}+\sqrt{(n-1)^{2}+(v+1)^{2}}$ with equality if and only if $G \cong H_{n, v}$.

We are now ready to prove one of the main results of this section.

Theorem 1. Let $G$ be a connected graph with a maximum value of Sombor index among all $n$-vertex connected graphs with cyclomatic number $v$ and assume that the number of pendent vertices is less than or equal to $n-v-2$. If $0 \leq v \leq n-2$, then $G \cong H_{n, v}$ and $S O(G)=$ $(n-v-2) \sqrt{(n-1)^{2}+1}+v \sqrt{(n-1)^{2}+4}+\sqrt{(n-1)^{2}+(v+1)^{2}}+v \sqrt{(v+1)^{2}+4}$.

Proof. By Lemma 3, we have $\Delta(G)=n-1$. Suppose $u \in V(G)$ and $d_{G}(u)=n-1$. By definition of Sombor index, $S O(G)=\sum_{v \in V(G) \backslash\{u\}} \sqrt{(n-1)^{2}+d_{G}(v)^{2}}+S O^{\ddagger}(G-u)$. Since $G-u$ is a graph of order $n-1$ with $v$ edges, Lemmas 4 and 6 imply that $S O(G)$ $\leq(n-v-2) \sqrt{(n-1)^{2}+1}+v \sqrt{(n-1)^{2}+4}+\sqrt{(n-1)^{2}+(v+1)^{2}}+v \sqrt{(v+1)^{2}+4}$, with equality if and only if $G \cong H_{n, v}$. This completes the proof of the theorem.

Lemma 7. If $G$ is an $n$-vertex graph with exactly $m$ edges, then

$$
S O(G) \leq m \sqrt{m^{2}+1} \text { and } S O_{r e d}(G) \leq m(m-1)
$$

with equalities if and only if $m \leq n-1$ and $G \cong S_{m+1} \cup(n-m-1) K_{1}$.

Proof. Suppose $u v \in E(G)$. Since $G$ is a graph with $m$ edges, $d_{G}(u)+d_{G}(v) \leq m+1$. On the other hand, the functions $f(x)=x^{2}$ and $g(x)=(x-1)^{2}$ are strictly convex on $(-\infty, \infty)$, and increasing on $[1, \infty)$. Therefore,

$$
\sqrt{d_{G}(u)^{2}+d_{G}(v)^{2}} \leq \sqrt{\left(d_{G}(u)+\lambda\right)^{2}+d_{G}(v)^{2}}
$$

and

$$
\sqrt{\left(d_{G}(u)-1\right)^{2}+\left(d_{G}(v)-1\right)^{2}} \leq \sqrt{\left(d_{G}(u)-1+\lambda\right)^{2}+\left(d_{G}(v)-1\right)^{2}},
$$

where $\lambda=m+1-\left(d_{G}(u)+d_{G}(v)\right)$. The equalities in (4) and (5) hold if and only if $d_{G}(u)+d_{G}(v)=m+1$. Furthermore, $\left(d_{G}(u)+\lambda, d_{G}(v)\right) \preceq(m, 1)$, and, by Lemma 1 , we obtain

$$
\sqrt{\left(d_{G}(u)+\lambda\right)^{2}+d_{G}(v)^{2}} \leq \sqrt{m^{2}+1}
$$

and

$$
\sqrt{\left(d_{G}(u)-1+\lambda\right)^{2}+\left(d_{G}(v)-1\right)^{2}} \leq(m-1) .
$$


The equalities in (6) and (7) hold if and only if $\left(d_{G}(u)+\lambda, d_{G}(v)\right)=(m, 1)$. Therefore, by (4)-(7),

$$
S O(G)=\sum_{u v \in E(G)} \sqrt{d_{G}(u)^{2}+d_{G}(v)^{2}} \leq \sum_{u v \in E(G)} \sqrt{m^{2}+1}=m \sqrt{m^{2}+1}
$$

and

$$
S O_{r e d}(G)=\sum_{u v \in E(G)} \sqrt{\left(d_{G}(u)-1\right)^{2}+\left(d_{G}(v)-1\right)^{2}} \leq \sum_{u v \in E(G)}(m-1)=m(m-1) .
$$

The equalities hold if and only if $m \leq n-1$ and $G \cong S_{m+1} \cup(n-m-1) K_{1}$, proving the lemma.

Corollary 1. If $T$ is an n-vertex tree, then

$$
S O(T) \leq(n-1) \sqrt{(n-1)^{2}+1} \text { and } S_{\text {red }}(T) \leq(n-1)(n-2) .
$$

The equalities hold if and only if $T \cong S_{n}$.

Lemma 8. Let $G$ be a graph with maximum reduced Sombor index among all connected graphs with $n$ vertices and cyclomatic number $v$. Then, $\Delta(G)=n-1$.

Proof. Suppose $v \in V(G), d_{G}(v)=\Delta(G), N(G, v)=\left\{x_{1}, x_{2}, \ldots, x_{\Delta}\right\}$ and $d_{G}(v) \leq n-2$. Since $G$ is a connected graph, there exists a positive integer $i, 1 \leq i \leq \Delta$, such that $A=N\left(G, x_{i}\right) \backslash(N(G, v) \cup\{v\}) \neq \varnothing$. Assume that $G^{\prime}=G-\left\{x_{i} a \mid a \in A\right\}+\{v a \mid a \in$ $A\}, B=N\left(G, x_{i}\right) \cap N(G, v), C=N(G, v) \backslash\left(B \cup\left\{x_{i}\right\}\right)$ and $\mu=S O_{\text {red }}(G)-S O_{\text {red }}\left(G^{\prime}\right)$. By definitions,

$$
\begin{aligned}
\mu= & \sum_{a \in A} \sqrt{\left(d_{G}(a)-1\right)^{2}+\left(d_{G}\left(x_{i}\right)-1\right)^{2}}+\sum_{b \in B} \sqrt{\left(d_{G}(b)-1\right)^{2}+\left(d_{G}\left(x_{i}\right)-1\right)^{2}} \\
& +\sum_{b \in B} \sqrt{\left(d_{G}(b)-1\right)^{2}+(\Delta-1)^{2}}+\sum_{c \in C} \sqrt{\left(d_{G}(c)-1\right)^{2}+(\Delta-1)^{2}} \\
& +\sqrt{\left(d_{G}\left(x_{i}\right)-1\right)^{2}+(\Delta-1)^{2}}-\left[\sum_{a \in A} \sqrt{\left(d_{G}(a)-1\right)^{2}+(\Delta+|A|-1)^{2}}\right. \\
& +\sum_{b \in B} \sqrt{\left(d_{G}(b)-1\right)^{2}+\left(d_{G}\left(x_{i}\right)-|A|-1\right)^{2}}+\sum_{b \in B} \sqrt{\left(d_{G}(b)-1\right)^{2}+(\Delta+|A|-1)^{2}} \\
& \left.+\sum_{c \in C} \sqrt{\left(d_{G}(c)-1\right)^{2}+(\Delta+|A|-1)^{2}}+\sqrt{\left(d_{G}\left(x_{i}\right)-|A|-1\right)^{2}+(\Delta+|A|-1)^{2}}\right] \\
& <\sum_{b \in B} \sqrt{\left(d_{G}(b)-1\right)^{2}+\left(d_{G}\left(x_{i}\right)-1\right)^{2}}+\sum_{b \in B} \sqrt{\left(d_{G}(b)-1\right)^{2}+(\Delta-1)^{2}} \\
& +\sqrt{\left(d_{G}\left(x_{i}\right)-1\right)^{2}+(\Delta-1)^{2}}-\left[\sum_{b \in B} \sqrt{\left(d_{G}(b)-1\right)^{2}+\left(d_{G}\left(x_{i}\right)-|A|-1\right)^{2}}\right. \\
& \left.+\sum_{b \in B} \sqrt{\left(d_{G}(b)-1\right)^{2}+(\Delta+|A|-1)^{2}}+\sqrt{\left(d_{G}\left(x_{i}\right)-|A|-1\right)^{2}+(\Delta+|A|-1)^{2}}\right]
\end{aligned}
$$


On the other hand, the functions $h_{1}(x)=\sqrt{\left(d_{G}(b)-1\right)^{2}+(x-1)^{2}}, b \in B$, and $h_{2}(x)=(x-1)^{2}$ are strictly convex on $(-\infty, \infty)$, and $\left(\Delta, d_{G}\left(x_{i}\right)\right) \prec\left(\Delta+|A|, d_{G}\left(x_{i}\right)-|A|\right)$. Apply Lemma 1 to obtain

$$
\begin{aligned}
& \sum_{b \in B} \sqrt{\left(d_{G}(b)-1\right)^{2}+\left(d_{G}\left(x_{i}\right)-1\right)^{2}}+\sum_{b \in B} \sqrt{\left(d_{G}(b)-1\right)^{2}+(\Delta-1)^{2}} \\
& +\sqrt{\left(d_{G}\left(x_{i}\right)-1\right)^{2}+(\Delta-1)^{2}}<\sum_{b \in B} \sqrt{\left(d_{G}(b)-1\right)^{2}+\left(d_{G}\left(x_{i}\right)-|A|-1\right)^{2}} \\
& +\sum_{b \in B} \sqrt{\left(d_{G}(b)-1\right)^{2}+(\Delta+|A|-1)^{2}}+\sqrt{\left(d_{G}\left(x_{i}\right)-|A|-1\right)^{2}+(\Delta+|A|-1)^{2}}
\end{aligned}
$$

Now, by (8) and (9), $S O_{\text {red }}(G)-S O_{\text {red }}\left(G^{\prime}\right)<0$, contradicted by the fact that $S O_{\text {red }}(G)$ is maximum. Thus, $\Delta(G)=n-1$. This completes the proof.

Lemma 9. Let $G$ be a connected graph of order $n(\geq 3)$ with cyclomatic number $v(0 \leq v \leq n-2)$ and vertex set $V(G)=\left\{v_{1}, v_{2}, \ldots, v_{n}\right\}$ with the condition that the number of pendent vertices is less than or equal to $n-v-2$. If $d(G)=\left(n-1, d_{G}\left(v_{2}\right), \ldots, d_{G}\left(v_{n}\right)\right)$, then

$$
\begin{gathered}
\sum_{v \in V(G) \backslash\left\{v_{1}\right\}} \sqrt{(n-2)^{2}+\left(d_{G}(v)-1\right)^{2}} \leq(n-v-2)(n-2)+v \sqrt{(n-2)^{2}+1} \\
+\sqrt{(n-2)^{2}+v^{2}}
\end{gathered}
$$

with equality if and only if $G \cong H_{n, v}$.

Proof. Suppose $g(x)=\sqrt{(n-2)^{2}+(x-1)^{2}}$. By Lemma 5, $g$ is strictly convex on $(-\infty, \infty)$. By Lemma $2,\left(d_{G}\left(v_{2}\right), d_{G}\left(v_{3}\right), \ldots, d_{G}\left(v_{n}\right)\right) \preceq(v+1,2, \ldots, 2,1, \ldots, 1)$, where the multiplicities of the numbers 1 and 2 in the last sequence are $n-v-2$ and $v$, respectively. By Lemma 1, we obtain

$$
\begin{aligned}
\sum_{v \in V(G) \backslash\left\{v_{1}\right\}} \sqrt{(n-2)^{2}+\left(d_{G}(v)-1\right)^{2}} \leq & (n-v-2)(n-2)+v \sqrt{(n-2)^{2}+1} \\
& +\sqrt{(n-2)^{2}+v^{2}}
\end{aligned}
$$

with equality if and only if $G \cong H_{n, v}$.

The following theorem is the second main result of this section.

Theorem 2. Suppose G has maximum reduced Sombor index among all n-vertex graphs with cyclomatic number $v$ and assume that the number of pendent vertices is less than or equal to $n-v-$ 2. If $0 \leq v \leq n-2$, then $G \cong H_{n, v}$ and $S O_{\text {red }}(G)=(n-v-2)(n-2)+v \sqrt{(n-2)^{2}+1}+$ $v \sqrt{v^{2}+1}+\sqrt{(n-2)^{2}+v^{2}}$.

Proof. By Lemma 8, we have $\Delta(G)=n-1$. Suppose $u \in V(G)$ and $d_{G}(u)=n-1$. By definition of a reduced Sombor index, $S O_{\text {red }}(G)=\sum_{v \in V(G) \backslash\{u\}} \sqrt{(n-2)^{2}+\left(d_{G}(v)-1\right)^{2}}+$ $S O(G-u)$. Since $G-u$ is a graph of order $n-1$ with $v$ edges, Lemmas 7 and 9 imply that $S O_{\text {red }}(G) \leq(n-v-2)(n-2)+v \sqrt{(n-2)^{2}+1}+v \sqrt{v^{2}+1}+\sqrt{(n-2)^{2}+v^{2}}$ with equality if and only if $G \cong H_{n, v}$.

The following lemma is useful in finding some new lower bounds for the Sombor and reduced Sombor indices of graphs. 
Lemma 10 (Ref. [21]). If $G$ is a graph with $n$ vertices, $m$ edges, and without isolated edges. Then, $\varepsilon_{1}(G)=4 m-M_{1}(G)+\sum_{i=3}^{2 n-4} \varepsilon_{i}(G)(i-2)$ and $\varepsilon_{2}(G)=M_{1}(G)-3 m-\sum_{i=3}^{2 n-4} \varepsilon_{i}(G)(i-1)$.

Suppose $x$ and $y$ are two positive real numbers and $x+y-2=s$. Since $x^{2}+y^{2}-$ $\frac{(s+2)^{2}}{2}=x^{2}+y^{2}-\frac{(x+y)^{2}}{2}=\frac{1}{2}(x-y)^{2}, x^{2}+y^{2} \geq \frac{(s+2)^{2}}{2}$, and the equality holds if and only if $x=y$. Thus, $\sqrt{x^{2}+y^{2}} \geq \frac{\sqrt{2}(s+2)}{2}$, and the equality holds if and only if $x=y$. We now give two lower bounds on Sombor index and reduced Sombor index for connected graph $G$ in terms of $m$ and the first Zagreb index.

Theorem 3. Let $G$ be a connected graph with $n \geq 3$ vertices and $m$ edges. Then, $S O(G)$ $\geq \frac{1}{3}(2 \sqrt{2}-\sqrt{5})\left(3 M_{1}(G)-4 m+2 \sqrt{5} \sqrt{2} m\right)$ and $S O_{r e d}(G) \geq(\sqrt{2}-1)\left(M_{1}(G)-2 m+\right.$ $\sqrt{2} m$ ). The equalities hold if and only if $G \cong P_{n}$ or $G \cong C_{n}$.

Proof. By definition of Sombor index,

$$
\begin{aligned}
S O(G) & =\sum_{1 \leq i \leq j \leq n-1} m_{i, j}(G) \sqrt{i^{2}+j^{2}} \\
& =m_{1,2} \sqrt{5}+m_{1,3} \sqrt{10}+m_{2,2} \sqrt{8}+\sum_{j=4}^{n-1} m_{1, j} \sqrt{1+j^{2}}+\sum_{j=3}^{n-1} m_{2, j} \sqrt{4+j^{2}} \\
& +\sum_{3 \leq i \leq j \leq n-1} m_{i, j} \sqrt{i^{2}+j^{2}}
\end{aligned}
$$

and by our discussion before the statement of this theorem,

$$
\begin{aligned}
S O(G) & \geq m_{1,2} \sqrt{5}+m_{1,3} \sqrt{10}+m_{2,2} \sqrt{8}+\sum_{j=4}^{n-1} m_{1, j} \frac{\sqrt{2}(j+1)}{2}+\sum_{j=3}^{n-1} m_{2, j} \frac{\sqrt{2}(j+2)}{2} \\
& +\sum_{3 \leq i \leq j \leq n-1} m_{i, j} \frac{\sqrt{2}(i+j)}{2} .
\end{aligned}
$$

On the other hand, $\sum_{1 \leq i \leq j \leq n-1} m_{i, j}-\left(m_{1,2}+m_{1,3}+m_{2,2}\right)=\sum_{i=3}^{2 n-4} \varepsilon_{i}(G)$. Therefore, by definition of $\varepsilon_{i}(G), 1 \leq i \leq 2 n-4$,

$$
\begin{aligned}
S O(G) & \geq m_{1,2} \sqrt{5}+m_{1,3} \sqrt{10}+m_{2,2} \sqrt{8}+\sum_{i=3}^{2 n-4} \varepsilon_{i}(G) \frac{\sqrt{2}(i+2)}{2} \\
& \geq m_{1,2} \sqrt{5}+\left(m_{1,3}+m_{2,2}\right) \sqrt{8}+\sum_{i=3}^{2 n-4} \varepsilon_{i}(G) \frac{\sqrt{2}(i+2)}{2} \\
& =\varepsilon_{1}(G) \sqrt{5}+\varepsilon_{2}(G) \sqrt{8}+\sum_{i=3}^{2 n-4} \varepsilon_{i}(G) \frac{\sqrt{2}(i+2)}{2}
\end{aligned}
$$

The equality holds if and only if $m=m_{1,2}+\sum_{i=2}^{n-1} m_{i, i}$. Now, by Lemma 10,

$$
\begin{aligned}
S O(G) & \geq\left[4 m-M_{1}(G)+\sum_{i=3}^{2 n-4} \varepsilon_{i}(G)(i-2)\right] \sqrt{5}+\sum_{i=3}^{2 n-4} \varepsilon_{i}(G) \frac{\sqrt{2}(i+2)}{2} \\
& +\left[M_{1}(G)-3 m-\sum_{i=3}^{2 n-4} \varepsilon_{i}(G)(i-1)\right] \sqrt{8} \\
& =\left[4 m-M_{1}(G)\right] \sqrt{5}+\left[M_{1}(G)-3 m\right] \sqrt{8}+\frac{1}{2} \sum_{i=3}^{2 n-4} \varepsilon_{i}(G)(2 \sqrt{5}-3 \sqrt{2})(i-2) \\
& \geq \frac{1}{3}(2 \sqrt{2}-\sqrt{5})\left(3 M_{1}(G)-4 m+2 \sqrt{5} \sqrt{2} m\right) .
\end{aligned}
$$


The equality holds if and only if $m=m_{1,2}+m_{2,2}$ that is, if and only if $G \cong P_{n}$ or $G \cong C_{n}$. By definition of a reduced Sombor index,

$$
\begin{aligned}
S O_{\text {red }}(G)= & \sum_{1 \leq i \leq j \leq n-1} m_{i, j}(G) \sqrt{(i-1)^{2}+(j-1)^{2}} \\
= & m_{1,2}+2 m_{1,3}+\sqrt{2} m_{2,2}+\sum_{j=4}^{n-1} m_{1, j} \sqrt{(j-1)^{2}}+\sum_{j=3}^{n-1} m_{2, j} \sqrt{1+(j-1)^{2}} \\
& +\sum_{3 \leq i \leq j \leq n-1} m_{i, j} \sqrt{(i-1)^{2}+(j-1)^{2}} .
\end{aligned}
$$

Using the same technique in the proof of first part, we obtain

$$
\begin{aligned}
S O_{\text {red }}(G) & \geq m_{1,2}+2 m_{1,3}+\sqrt{2} m_{2,2}+\sum_{i=3}^{2 n-4} \varepsilon_{i}(G) \frac{\sqrt{2} i}{2} \\
& \geq m_{1,2}+\sqrt{2}\left(m_{1,3}+m_{2,2}\right)+\sum_{i=3}^{2 n-4} \varepsilon_{i}(G) \frac{\sqrt{2} i}{2} \\
& =\varepsilon_{1}(G)+\sqrt{2} \varepsilon_{2}(G)+\sum_{i=3}^{2 n-4} \varepsilon_{i}(G) \frac{\sqrt{2} i}{2} .
\end{aligned}
$$

The equality holds if and only if $m=m_{1,2}+\sum_{i=2}^{n-1} m_{i, i}$. Now, by Lemma 10,

$$
\begin{aligned}
S O_{\text {red }}(G) \geq & {\left[4 m-M_{1}(G)+\sum_{i=3}^{2 n-4} \varepsilon_{i}(G)(i-2)\right]+\sum_{i=3}^{2 n-4} \varepsilon_{i}(G) \frac{\sqrt{2} i}{2} } \\
& +\sqrt{2}\left[M_{1}(G)-3 m-\sum_{i=3}^{2 n-4} \varepsilon_{i}(G)(i-1)\right] \\
\geq & {\left[4 m-M_{1}(G)\right]+\sqrt{2}\left[M_{1}(G)-3 m\right]+\frac{1}{2} \sum_{i=3}^{2 n-4} \varepsilon_{i}(G)(2-\sqrt{2})(i-2) } \\
\geq & (\sqrt{2}-1)\left(M_{1}(G)-2 m+\sqrt{2} m\right) .
\end{aligned}
$$

The equality holds if and only if $m=m_{1,2}+m_{2,2}$, that is, if and only if $G \cong P_{n}$ or $G \cong C_{n}$.

\section{The Case of $v=6$}

The aim of this section is to prove the case of $v=6$ in Conjecture 1 . In addition, we give some information on the structure of the graphs with maximum Sombor index (resp. reduced Sombor index) among connected $n$-vertex $v$-cyclic graphs. To do this, let $\mathbb{E} \mathbb{S}$ be the set of graphs $G$ of order at least 1 satisfying: for any vertices $v$ and $v^{\prime}$ of $G$, if $d_{G}(v) \leq d_{G}\left(v^{\prime}\right)$, then $N(G, v)$ is a subset of $N\left(G, v^{\prime}\right) \cup\left\{v^{\prime}\right\}$. In addition, assume that $\mathbb{G}(n, v)$ denotes the set of all graphs with exactly $n$ vertices and cyclomatic number $v$, where $0 \leq v \leq n-2$. If $G \in \mathbb{G}(n, v)$, then we use that notation $n_{1}(G)$ for the number of pendent vertices of $G$. If $0 \leq v \leq n-2$, then we define:

$$
\begin{aligned}
& \Omega_{1}^{n, v}=\{G \in \mathbb{G}(n, v) \mid \Delta(G) \leq n-2\}, \\
& \Omega_{2}^{n, v}=\left\{G \in \mathbb{G}(n, v) \mid \Delta(G)=n-1 \text { and } n_{1}(G) \leq n-v-2\right\}, \\
& \Omega_{3}^{n, v}=\left\{G \in \mathbb{G}(n, v) \mid \Delta(G)=n-1, n_{1}(G) \geq n-v-1 \text { and } G \notin \mathbb{E} S\right\}, \\
& \Omega_{4}^{n, v}=\left\{G \in \mathbb{G}(n, v) \mid \Delta(G)=n-1, n_{1}(G) \geq n-v-1 \text { and } G \in \mathbb{E} \mathbb{S}\right\},
\end{aligned}
$$

It is easy to see that $\left\{\Omega_{1}^{n, v}, \Omega_{2}^{n, v}, \Omega_{3}^{n, v}, \Omega_{4}^{n, v}\right\}$ is a partition for $\mathbb{G}(n, v)$. 
Lemma 11. Let $G$ be a graph with a maximum Sombor index among connected graphs with $n$ vertices and cyclomatic number $v$. Then, $\Delta(G)=n-1$.

The proof of Lemma 11 is similar to that of Lemma 8, so we omit it.

Lemma 12. Let $G$ be a graph with maximum Sombor index (resp. reduced Sombor index) among connected n-vertex $v$-cyclic graphs. Then, $G$ is in $\mathbb{E} \mathbb{S}$.

Proof. We prove this result by contradiction. For this, we assume that $u, v \in V(G)$, $d_{G}(u) \leq d_{G}(v)$ and $N(G, u) \backslash(N(G, v) \cup\{v\})=\left\{u_{1}, \ldots, u_{r}\right\}, r \geq 1$. Assume that $G^{\prime}=G-\left\{u u_{i} \mid i=1, \ldots, r\right\}+\left\{v u_{i} \mid i=1, \ldots, r\right\}, A=N(G, u) \cap N(G, v), \Lambda=S O(G)-$ $S O\left(G^{\prime}\right)$ and $Y=S O_{\text {red }}(G)-S O_{\text {red }}\left(G^{\prime}\right)$. By Lemmas 8 and $11, G^{\prime}$ is a connected $n$ vertex $v$-cyclic graph. Now, via the majorization technique, one can easily check that $\sqrt{d_{G}(u)^{2}+d_{G}(v)^{2}}<\sqrt{\left(d_{G}(u)-r\right)^{2}+\left(d_{G}(v)+r\right)^{2}}$ and $\sqrt{\left(d_{G}(u)-1\right)^{2}+\left(d_{G}(v)-1\right)^{2}}<$ $\sqrt{\left(d_{G}(u)-r-1\right)^{2}+\left(d_{G}(v)+r-1\right)^{2}}$. Therefore, by definitions,

$$
\begin{aligned}
\Lambda \leq & \sum_{i=1}^{r} \sqrt{d_{G}(u)^{2}+d_{G}\left(u_{i}\right)^{2}}+\sum_{a \in A} \sqrt{d_{G}(u)^{2}+d_{G}(a)^{2}}+\sum_{a \in A} \sqrt{d_{G}(v)^{2}+d_{G}(a)^{2}} \\
& -\left[\sum_{i=1}^{r} \sqrt{\left(d_{G}(v)+r\right)^{2}+d_{G}\left(u_{i}\right)^{2}}+\sum_{a \in A} \sqrt{\left(d_{G}(u)-r\right)^{2}+d_{G}(a)^{2}}\right. \\
& \left.+\sum_{a \in A} \sqrt{\left(d_{G}(v)+r\right)^{2}+d_{G}(a)^{2}}\right], \\
\mathrm{Y} \leq & \sum_{i=1}^{r} \sqrt{\left(d_{G}(u)-1\right)^{2}+\left(d_{G}\left(u_{i}\right)-1\right)^{2}}+\sum_{a \in A} \sqrt{\left(d_{G}(u)-1\right)^{2}+\left(d_{G}(a)-1\right)^{2}} \\
& +\sum_{a \in A} \sqrt{\left(d_{G}(v)-1\right)^{2}+\left(d_{G}(a)-1\right)^{2}}-\left[\sum_{i=1}^{r} \sqrt{\left(d_{G}(v)+r-1\right)^{2}+\left(d_{G}\left(u_{i}\right)-1\right)^{2}}\right. \\
& \left.+\sum_{a \in A} \sqrt{\left(d_{G}(u)-r-1\right)^{2}+\left(d_{G}(a)-1\right)^{2}}+\sum_{a \in A} \sqrt{\left(d_{G}(v)+r-1\right)^{2}+\left(d_{G}(a)-1\right)^{2}}\right] .
\end{aligned}
$$

The equalities hold if and only if $u v \notin E(G)$. On the other hand,

$$
\begin{gathered}
\sum_{i=1}^{r} \sqrt{d_{G}(u)^{2}+d_{G}\left(u_{i}\right)^{2}}<\sum_{i=1}^{r} \sqrt{\left(d_{G}(v)+r\right)^{2}+d_{G}\left(u_{i}\right)^{2}}, \\
\sum_{i=1}^{r} \sqrt{\left(d_{G}(u)-1\right)^{2}+\left(d_{G}\left(u_{i}\right)-1\right)^{2}}<\sum_{i=1}^{r} \sqrt{\left(d_{G}(v)+r-1\right)^{2}+\left(d_{G}\left(u_{i}\right)-1\right)^{2}} .
\end{gathered}
$$

The functions $h_{1}(x)=\sqrt{x^{2}+d_{G}(a)^{2}}$ and $h_{2}(x)=\sqrt{(x-1)^{2}+\left(d_{G}(a)-1\right)^{2}}, a \in A$, are strictly convex on $(-\infty, \infty)$, and $\left(d_{G}(v), d_{G}(u)\right) \prec\left(d_{G}(v)+r, d_{G}(u)-r\right)$. By Lemma 1 , from (10) and (11), we obtain $S O(G)-S O\left(G^{\prime}\right)<0$ and $S O_{\text {red }}(G)-S O_{\text {red }}\left(G^{\prime}\right)<0$, contradicted by the fact that $S O(G)$ (resp. $S O_{\text {red }}(G)$ ) is maximum. Thus, $G$ is in $\mathbb{E} \mathbb{S}$. This completes the proof.

Lemma 13. Let $G$ be a graph in $\mathbb{E} S$ of order at least 2. Then, $G$ has an isolated or universal vertex $u$, with $G-\{u\}$ being in $\mathbb{E} \mathbb{S}$.

Proof. Let $G$ be in $\mathbb{E} \mathbb{S}$. Assume that $G$ has no isolated vertex and let $v$ be a vertex of $G$ with a maximum degree, and let $u \in V(G) \backslash\{v\}$ and $w \in N(G, u)$. As $G$ is in $\mathbb{E} \mathbb{S}$ and $d_{G}(w) \leq d_{G}(v), N(G, w) \subseteq N(G, v) \cup\{v\}$. Thus, $u v \in E(G)$, and therefore $v$ is universal vertex in $G$. It is easy to check that, if $u$ is isolated or universal, then $G-\{u\}$ is in $\mathbb{E} \mathbb{S}$.

The following characterization of $\mathbb{E} \mathbb{S}$ gives some information on the structure of the graphs with maximum Sombor index (resp. reduced Sombor index) among connected $n$-vertex $v$-cyclic graphs. 
Lemma 14. The following statements hold:

(1) $K_{1}$ is in $\mathbb{E S}$,

(2) if $G$ is in $\mathbb{E} \mathbb{S}$, then $G \cup K_{1}$ is in $\mathbb{E} \mathbb{S}$,

(3) if $G$ is in $\mathbb{E} \mathbb{S}$, then $G+K_{1}$ is in $\mathbb{E} \mathbb{S}$,

(4) each graph of $\mathbb{E} \mathbb{S}$ is obtained from $K_{1}$ by applying rules 2) and 3) a finite number of times.

Proof. If $G$ is in $\mathbb{E} \mathbb{S}$, then it is easy to see that $G \cup K_{1}$ and $G+K_{1}$ is in $\mathbb{E} \mathbb{S}$. In addition, by induction on order of graph and using Lemma 13, one can easily check that any graph in $\mathbb{E} \mathbb{S}$ is obtained from $K_{1}$ by applying rules (2) and (3) a finite number of times.

Theorem 4. Let $G \in \mathbb{G}(n, v)$. If $G$ has a maximum Sombor index or a reduced Sombor index, then $G \in \Omega_{4}^{n, v}$ or $G \cong H_{n, v}$.

Proof. The proof follows from Lemmas 3, 8 and 12 and Theorems 1 and 2.

In the following theorem, it is proved that Conjecture 1 for the case that $v=6$ is correct.

Theorem 5. Let $n$ is a positive integer greater than or equal to 8 . Then, the graph $H_{n, 6}$ has maximum $S O$ and $S O_{\text {red }}$ among all n-vertex connected graphs with cyclomatic number 6.

Proof. Suppose $G_{1}, G_{2}$ and $G_{3}$ are graphs which are depicted in Figure 1. In addition, suppose $F_{1}=\left(G_{1} \cup(n-5) K_{1}\right)+K_{1}, F_{2}=\left(G_{2} \cup(n-6) K_{1}\right)+K_{1}$, and $F_{3}=\left(G_{3} \cup(n-\right.$ 7) $\left.K_{1}\right)+K_{1}$. By Lemma $14, \Omega_{4}^{n, 6}=\left\{F_{1}, F_{2}, F_{3}\right\}$. Now, by the structures of the graphs $F_{1}, F_{2}$ and $F_{3}$, it is easy to see that

$$
\begin{aligned}
& S O\left(H_{n, 6}\right)-S O\left(F_{1}\right) \\
& =\sqrt{(n-1)^{2}+49}-4 \sqrt{(n-1)^{2}+16}+6 \sqrt{53}-24 \sqrt{2}-3 \sqrt{(n-1)^{2}+1}+6 \sqrt{(n-1)^{2}+4} \\
& >7 \sqrt{(n-1)^{2}+4}-7 \sqrt{(n-1)^{2}+16}+6 \sqrt{53}-24 \sqrt{2} \\
& =-\frac{84}{\sqrt{(n-1)^{2}+4}+\sqrt{(n-1)^{2}+16}}+6 \sqrt{53}-24 \sqrt{2}>4, \\
& S O_{\text {red }}\left(H_{n, 6}\right)-S O_{\text {red }}\left(F_{1}\right) \\
& =6 \sqrt{37}+6 \sqrt{(n-2)^{2}+1}+\sqrt{(n-2)^{2}+36}-4 \sqrt{(n-2)^{2}+9}-18 \sqrt{2}-3 \sqrt{(n-2)^{2}} \\
& >6 \sqrt{37}+7 \sqrt{(n-2)^{2}+1}-18 \sqrt{2}-7 \sqrt{(n-2)^{2}+9}>6 \text {, } \\
& S O\left(H_{n, 6}\right)-S O\left(F_{2}\right)>5 \sqrt{(n-1)^{2}+4}-5 \sqrt{(n-1)^{2}+16}+6 \sqrt{53}-10-\sqrt{29}-2 \sqrt{34}-\sqrt{41}>6, \\
& S O_{\text {red }}\left(H_{n, 6}\right)-S O_{\text {red }}\left(F_{2}\right)>5 \sqrt{(n-2)^{2}+1}-5 \sqrt{(n-2)^{2}+9}-\sqrt{17}-2 \sqrt{13}+6 \sqrt{37}-4 \sqrt{5}-5>8, \\
& S O\left(H_{n, 6}\right)-S O\left(F_{7}\right)>3 \sqrt{(n-1)^{2}+4}-3 \sqrt{(n-1)^{2}+9}-6 \sqrt{5}+6 \sqrt{53}-3 \sqrt{2}-6 \sqrt{2} \sqrt{5}>6, \\
& S O_{\text {red }}\left(H_{n, 6}\right)-S O_{\text {red }}\left(F_{7}\right)>3 \sqrt{(n-2)^{2}+1}-3 \sqrt{(n-2)^{2}+4}-2 \sqrt{29}-3 \sqrt{26}-2 \sqrt{2}+6 \sqrt{37}>6 .
\end{aligned}
$$

Therefore, $S O\left(F_{i}\right)<S O\left(H_{n, 6}\right)$ and $S O_{\text {red }}\left(F_{i}\right)<S O_{\text {red }}\left(H_{n, 6}\right)$ for $i \in\{1,2,3\}$. Thus, the proof follows from Theorem 4.

A similar argument as Theorem 5 shows that the graph $H_{n, v}, 0 \leq v \leq 5$, has the maximum reduced Sombor index among all connected $n-$ vertex graphs, $n \geq v-2$. By Theorem 4, Conjecture 1 can be reduced to the following conjecture:

Conjecture 2. Suppose $n$ and $v$ are non-negative integers such that $7 \leq v \leq n-2$. If $G \in \Omega_{4}^{n, v}$, then $S O(G)<S O\left(H_{n, v}\right)$ and $S O_{\text {red }}(G)<S O_{\text {red }}\left(H_{n, v}\right)$. 


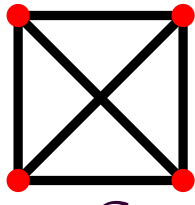

G1
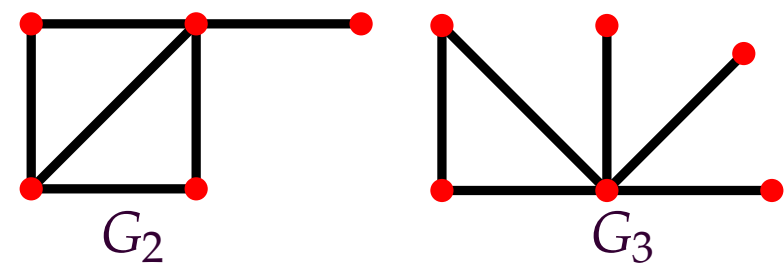

Figure 1. All $n$-vertex connected graphs in $\mathbb{E S}, n \leq 6$, with exactly six edges.

\section{Conclusions}

In this paper, the authors develop a method to calculate the maximum value of the Sombor index of graphs. This method is applied to partially solve a conjecture of Réti et al. [20]. In an exact phrase, it is proved that the graph $H_{n, 6}$ has the maximum Sombor index among all connected 6-cyclic graphs of order $n$. Moreover, we proved that the graph $H_{n, v}$ has the maximum Sombor index among all connected $v$-cyclic graphs of order $n$, where $5 \leq v \leq n-2$ and the number of pendent vertices is less than or equal to $n-v-2$. It is also proved that, under the same conditions, this conjecture is valid for the reduced Sombor index.

Author Contributions: Investigation, K.C.D., A.G. and A.R.A.; writing-original draft preparation, K.C.D., A.G. and A.R.A.; writing-review and editing, K.C.D., A.G. and A.R.A. All authors have read and agreed to the published version of the manuscript.

Funding: This research received no external funding.

Institutional Review Board Statement: Not applicable.

Informed Consent Statement: Not applicable.

Data Availability Statement: Not applicable.

Acknowledgments: The authors are grateful to the referees for their valuable comments and suggestions.

Conflicts of Interest: The authors declare that there is no conflict of interest.

\section{References}

1. West, D.B. Introduction to Graph Theory, 2nd ed.; Prentice Hall: Hoboken, NJ, USA, 2001.

2. Gutman, I.; Trinajstić, N. Graph theory and molecular orbitals, Total $\pi$-electron energy of alternant hydrocarbons. Chem. Phys. Lett. 1972, 17, 535-538. [CrossRef]

3. Gutman, I. Geometric approach to degree-based topological indices: Sombor indices. MATCH Commun. Math. Comput. Chem. 2021, 86, 11-16.

4. Redžepović, I. Chemical applicability of Sombor indices. J. Serb. Chem. Soc. 2021, 86, 445-457.

5. Rada, J.; Bermudo, S. Is every graph the extremal value of a vertex-degree-based topological index? MATCH Commun. Math. Comput. Chem. 2019, 81, 315-323.

6. Yao, Y.; Liu, M.; Das, K.C.; Ye, Y. Some extremal results for vertex-degree-Based invariants. MATCH Commun. Math. Comput. Chem. 2019, 81, 325-344.

7. Liu, M.; Liu, B.; Das, K.C. Recent results on the majorization theory of graph spectrum and topological index theory-A survey. Electron. J. Linear Algebra 2015, 30, 402-421. [CrossRef]

8. Marshall, A.W.; Olkin, I. Inequalities: Theory of Majorization and Its Applications; Academic Press: New York, NY, USA, 1979.

9. Gutman, I. Some basic properties of Sombor indices. Open J. Discret. Appl. Math. 2021, 4, 1-3. [CrossRef]

10. Cruz, R.; Gutman, I.; Rada, J. Sombor index of chemical graphs. Appl. Math. Comput. 2021, 399, 126018.

11. Cruz, R.; Rada, J. Extremal values of the Sombor index in unicyclic and bicyclic graphs. J. Math. Chem. 2021, 59, 1098-1116. [CrossRef]

12. Das, K.C.; Cevik, A.S.; Cangul, I.N.; Shang, Y. On Sombor index. Symmetry 2021, 13, 140. [CrossRef]

13. Deng, H.; Tang, Z.; Wu, R. Molecular trees with extremal values of Sombor indices. Int. J. Quantum Chem. 2021, 121, e26622,

14. Milovanović, I.; Milovanović, E.; Matejić, M. On some mathematical properties of Sombor indices. Bull. Int. Math. Virtual Inst. 2021, 11, 341-353.

15. Wang, Z.; Mao, Y.; Li, Y.; Furtula, B. On relations between Sombor and other degree-based indices. J. Appl. Math. Comput. 2021, 1-17. [CrossRef]

16. Das, K.C.; Shang, Y. Some extremal graphs with respect to Sombor index. Mathematics 2021, 9, 1202. [CrossRef] 
17. Das, K.C.; Gutman, I. On Sombor index of trees. Appl. Math. Comput. 2022, 412, 126575.

18. Ghanbari, N.; Alikhani, S. Sombor index of certain graphs. Iranian J. Math. Chem. 2021, 12, 27-37.

19. Hardy, G.H.; Littlewood, J.E.; Polya, G. Inequalities; Cambridge University Press: Cambridge, UK, 1952.

20. Réti, T.; Došlić, T.; Ali, A. On the Sombor index of graphs. Contrib. Math. 2021, 3, 11-18.

21. Ghalavand, A.; Ashrafi, A.R. Bounds on the entire Zagreb indices of graphs. MATCH Commun. Math. Comput. Chem. 2019, $81,371-381$. 